\title{
Droplet formation in microfluidic T-junction generators operating in the transitional regime. II. Modeling
}

\author{
Tomasz Glawdel, Caglar Elbuken, and Carolyn L. Ren* \\ Department of Mechanical and Mechatronics Engineering, University of Waterloo, Waterloo, Ontario, Canada N2L 3G1
}

(Received 12 October 2011; published 26 January 2012)

\begin{abstract}
This is the second part of a two-part study on the generation of droplets at a microfluidic T-junction operating in the transition regime. In the preceding paper [Phys. Rev. E 85, 016322 (2012)], we presented our experimental observations of droplet formation and decomposed the process into three sequential stages defined as the lag, filling, and necking stages. Here we develop a model that describes the performance of microfluidic T-junction generators working in the squeezing to transition regimes where confinement of the droplet dominates the formation process. The model incorporates a detailed geometric description of the drop shape during the formation process combined with a force balance and necking criteria to define the droplet size, production rate, and spacing. The model inherently captures the influence of the intersection geometry, including the channel width ratio and height-to-width ratio, capillary number, and flow ratio, on the performance of the generator. The model is validated by comparing it to speed videos of the formation process for several T-junction geometries across a range of capillary numbers and viscosity ratios.
\end{abstract}

DOI: 10.1103/PhysRevE.85.016323

PACS number(s): 47.61.Jd, 47.55.db

\section{INTRODUCTION}

In the preceding paper we presented our experimental observations on the operation of T-junction generators in the transitional regime [1]. The T-junction configuration is one of the most versatile designs for generating droplets in microfluidic devices [2]. The T-junction geometries are characterized by the width ratio $\Lambda^{*}=w_{d} / w_{c}$ of the dispersed and continuous phase channels and the height-to-width ratio of the microchannels $h^{*}=h / w_{c}$ (refer to Fig. 1). The operational mode of the generator is characterized by the capillary number $\mathrm{Ca}=\mu U / \gamma$ (with $U$ and $\mu$ the average velocity and viscosity of the continuous phase, respectively, and $\gamma$ the interfacial tension), which expresses the relative importance of viscous and capillary forces. At modest $\mathrm{Ca}$, T-junction generators operate in a transitional regime where confinement of the emerging interface creates a buildup of pressure that shears off droplets [3]. Our analysis of high-speed videos indicated that the formation process could be divided into three sequential stages: (i) a lag stage immediately after detachment of the previous droplet where the interface recedes back into the side channel, (ii) a filling stage where the droplet penetrates into the cross-flowing stream and hydrodynamic forces deform the droplet, and (iii) a necking stage where forces overwhelm the droplet, leading to pinchoff of the droplet (see Fig. 1). We identified several key parameters that describe the evolution of the droplet including the lag distance $L_{\text {lag }}$ after detachment, the penetration depth at the end of the filling stage $b_{\text {fill }}$, the necking stage $b_{\text {pinch }}$, and the critical neck thickness at pinchoff $2 r_{\text {pinch. }}$. In the preceding paper we analyzed the dependence of these parameters on geometric $\left(\Lambda^{*}, h^{*}\right)$ and flow conditions (Ca and the flow ratio $\varphi=Q_{d} / Q_{c}$ ).

In this paper we seek to develop a robust model that describes the entire operation of a T-junction generator in the transition regime so that one can predict the size of droplets formed, the spacing between droplets, and the frequency of

\footnotetext{
*c3ren@uwaterloo.ca
}

formation. An analysis of the three stages suggests that the scaling law that describes the size of the droplets has the form

$$
\frac{V_{\text {drop }}}{w_{c}^{2} h}=\alpha_{\mathrm{lag}}+\alpha_{\mathrm{fill}}+\beta \varphi,
$$

where the volume is normalized by $w_{c}^{2} h, \alpha_{\text {lag }}$ is the volume added during the lag stage, $\alpha_{\text {fill }}$ is the filling stage, and $\beta$ is the dimensionless necking time. These parameters are generally obtained through empirical fitting; however, our goal in this study is to develop mathematical expressions for these three parameters based on theoretical arguments.

The model formulation begins with a general geometric description of the three-dimensional (3D) shape of the droplet throughout the formation process. This geometric description is used to calculate the evolution of the volume of the droplet during the three stages of the formation process. First, the contribution of the lag stage $\alpha_{\text {lag }}$ is calculated as the volume added as the interface recovers in the side channel. Second, the filling volume $\alpha_{\text {fill }}$ is calculated, which includes a different force balance that predicts the penetration depth and includes viscosity and geometric influences. Third, the necking parameter is calculated using a control volume analysis, which also includes an alternative criterion for pinchoff based on our own experimental observations in the transition regime. In addition to the droplet volume, equations are derived for the spacing and frequency so that the entire operation of the generator is defined. The predictive capabilities of the model are verified by performing a detailed experimental analysis to validate the model across several different $\mathrm{T}$-junction designs and flow conditions.

\section{MODEL DEVELOPMENT}

\section{A. Model limits and constraints}

The model developed herein applies to droplet formation in the squeezing-to-transition regime where the breakup process is governed by the squeezing pressure generated as the interface extends into the cross-flowing stream [3-5]. Fluid 


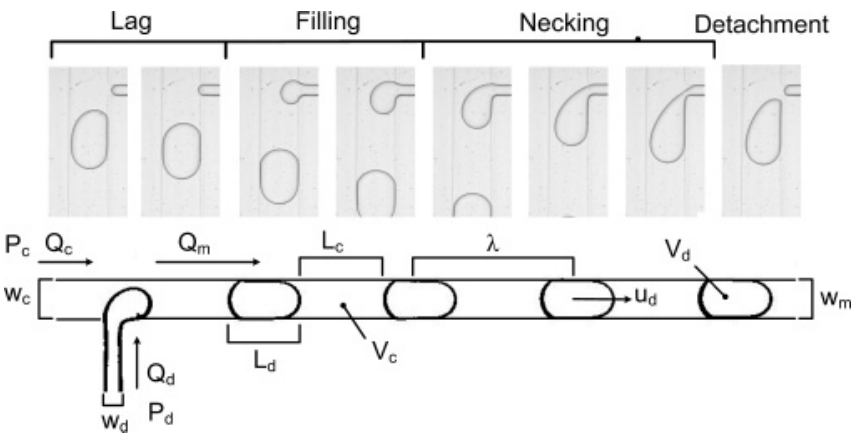

FIG. 1. Shown on top is the droplet formation cycle in the Tjunction generator consisting of three stages: the lag, filling, and necking periods. Shown on bottom is the global overview of the Tjunction generator with the respective geometric and flow parameters identified. Definitions include the flow rates $Q$, pressures $P$, and widths $w$ of the continuous, dispersed, and main channels and the height $h$, which is uniform throughout the network. Droplets are formed with a length $L_{d}$ and volume $V_{d}$, at a frequency $f$ and spacing $\lambda$, and are transported along the channel at a velocity $u_{d}$. The space between the droplets occupied by the continuous fluid is $L_{c}$ and has a volume $V_{c}$. Several dimensionless terms define the operation of the generator, including the width ratio $\Lambda^{*}=w_{d} / w_{c}$, height-towidth ratio $h^{*}=h / w_{c}$, flow ratio $\varphi=Q_{d} / Q_{c}$, and viscosity ratio $\eta=\mu_{d} / \mu_{c}$.

flow is laminar and creeping with $\mathrm{Re}<1$ so that inertia forces are negligible and buoyancy forces may also be ignored.

Droplets grow large enough to fill the entire cross section of the channel and form plugs that are longer than the channel width. Droplets are nonwetting and their shape is assumed to be in a state of quasiequilibrium that attempts to minimize the surface energy throughout the formation process. Marangoni stresses and dynamic interfacial effects are neglected in the following analysis, so it is assumed that the interfacial tension does not change during the formation process and is equal to the equilibrium value. This is valid when surfactants are absent from the system, as is the case for the experiments performed in this study, or when adsorption kinetics are fast compared to the formation process [6,7].

The confined geometry stabilizes the neck filament against perturbations such that the mechanism for pinchoff is not the growth of capillary instabilities, as is the case for unconfined geometries [8], but the reversal of flow around the drop due to Laplace pressure changes as the droplet shape evolves $[9,10]$. Only the condition where the two flow rates are relatively steady during the entire formation process is considered, thus the following model is applicable to both syringe pump and pressure driven flow as long as this condition is met. The geometry we consider is limited to cases where $h \leqslant w_{d}$ and $w_{c}$. In the following discussions, we denote dimensionless values by an asterisk, where lengths are normalized by $w_{c}$ and volumes by $w_{c}^{2} h$.

\section{B. Geometric description of droplet}

The general description of the shape of the droplet during the formation cycle is shown in Fig. 2, which follows the description provided by van Steijn et al. but modified into a more general form applicable to the transition regime [11].

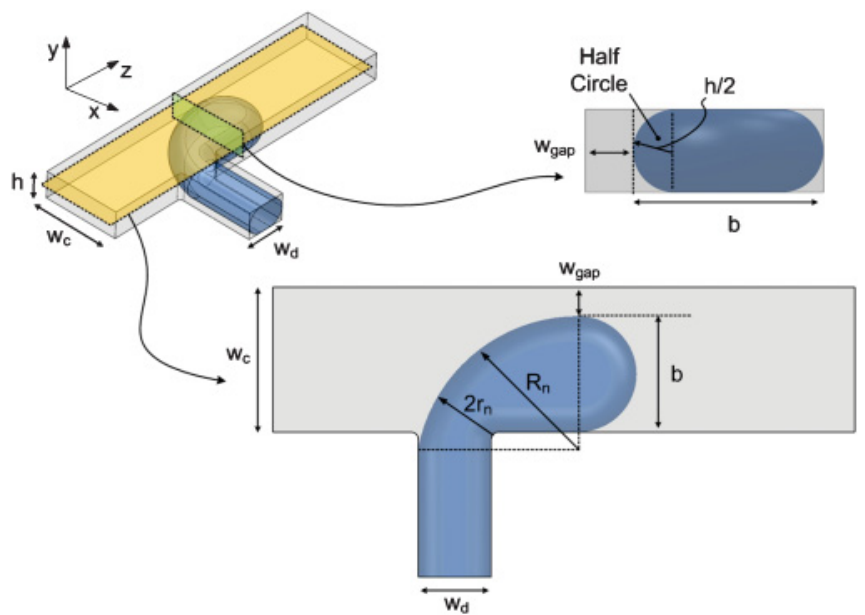

FIG. 2. (Color online) Geometric reconstruction of the droplet shape as it is being formed in the transition regime.

From the 2D planar view of the droplet, the $3 \mathrm{D}$ shape is reconstructed by assuming that the interface is confined by the upper and bottom walls and that it attempts to minimize surface energy so that the out-of-plane curvature is $h / 2$. The interface penetrates a distance $b$ into the main channel. The difference between the far channel wall and the interface is the gap width $w_{\text {gap }}=\left(w_{c}-b\right)$. A half circle of diameter $b$ defines the front half of the droplet, while the back is defined by a circle of radius $R_{n}$. The shortest distance from the corner of the T-junction to the back of the droplet is the neck thickness given as $2 r_{n}$.

The nondimensionalized volume of the droplet can be estimated from the $2 \mathrm{D}$ shape by projecting the area $A_{p}{ }^{*}$ through the channel depth and then including the out-of-plane curvature along the perimeter $l^{*}=l / w_{c}$ :

$$
V^{*}=A_{p}^{*} \pm\left(\frac{h^{*}}{2}\right)\left(1-\frac{\pi}{4}\right) 1^{*} .
$$

A negative sign is used in the second term for the dispersed phase, while a positive sign is used for the continuous phase. This geometric description serves as the basis for deriving expressions for the droplet volume and neck shape during the formation process.

\section{Lag stage}

Immediately after a drop detaches the interface recedes back into the side channel to a distance $L_{\text {lag }}$. Once the interface recovers it proceeds forward until it reaches the entrance of the channel (see Fig. 1). The volume of this recovery represents the portion of the total volume that is contributed by the lag stage.

Based on the geometric representation presented in Fig. 3 and the application of Eq. (2), the dimensionless lag volume is given by

$$
V_{\text {lag }}^{*}=\alpha_{\text {lag }}=L_{\text {lag }}^{*} \Lambda^{*}+\frac{1}{2}\left(1-\frac{\pi}{4}\right)\left(\left(\Lambda^{*}\right)^{2}+\frac{\pi \Lambda^{*} h^{*}}{2}\right),
$$

where $\Lambda^{*}=w_{d} / w_{c}$ is the width ratio and $h^{*}=h / w_{c}$ is the height aspect ratio. Experimental observations showed that 


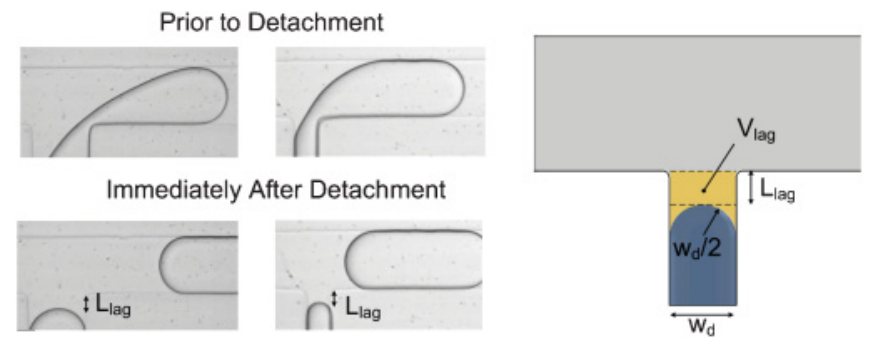

FIG. 3. (Color online) Interface shape immediately after detachment where it recedes back into the dispersed phase inlet. The position of the interface relative to the main channel is defined by the lag length $L_{\text {lag }}$ and the volume is defined by $V_{\text {lag }}$.

$L_{\text {lag }}$ is a complicated relationship between the flow conditions and geometry [1]. An adequate description is not available at the moment for $L_{\mathrm{lag}}$; thus, in the present model, $L_{\mathrm{lag}}$ is taken directly from experimental measurements and substituted into Eq. (3).

\section{Filling stage}

The filling stage ends when forces on the droplet balance and the neck of the droplet starts to collapse toward the inner corner of the T-junction. In concurrence with observations made by Christopher et al. and van Steijn et al., our own interpretation of the experimental evidence also advocates that the final size of the droplet at the end of the filling stage has the characteristic shape described in Fig. 4 [3,11]. Again, the front half of the droplet is defined by a half circle of diameter $b_{\text {fill }}$ and

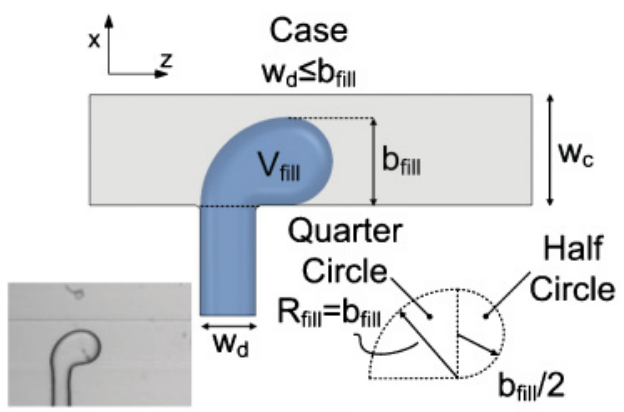

(a)

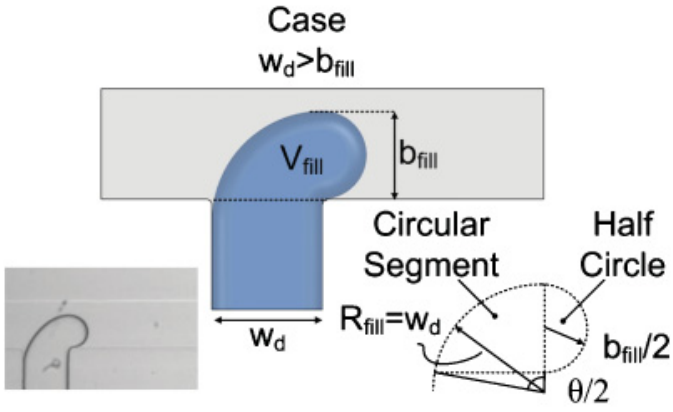

(b)

FIG. 4. (Color online) Droplet shape at the end of the filling stage for two cases where $w_{d} \leqslant b_{\text {fill }}$ and $w_{d}>b_{\text {fill }}$. The drops are characterized by the extent of the protrusion $b_{\text {fill }}$ and the neck radius at the filling stage $R_{\text {fill }}$. For the circular segment, the height is given by $b$ and the radius of the circle is $w_{d}$. the back half is defined by a quarter circle of diameter $R_{\text {fill }}$. Two cases exist depending on the geometry of the T-junction: the neck radius is confined (i) by a virtual channel wall created by the interface at $b_{\text {fill }}$ or (ii) by the inlet channel. This condition is defined as $R_{\text {fill }}=\max \left(w_{d}, b_{\text {fill }}\right)$.

The final volume at the end of the filling stage can be calculated by applying Eq. (2) to the two shapes. For $w_{d} \leqslant b_{\text {fill }}$ the result is

$\alpha_{\text {fill }}=\frac{3}{8} \pi\left(b_{\text {fill }}^{*}\right)^{2}-\frac{h^{*}}{2}\left(1-\frac{\pi}{4}\right)\left[\pi b_{\text {fill }}^{*}+\left(b_{\text {fill }}^{*}-\Lambda^{*}\right)\right]$.

For $w_{d}>b_{\text {fill }}$, the calculation is slightly different as the back part is defined by a half circular segment [12]:

$$
\begin{aligned}
\alpha_{\text {fill }}= & \frac{\pi}{8}\left(b_{\text {fill }}^{*}\right)^{2}+\frac{1}{4}\left(\Lambda^{*}\right)^{2}(\theta-\sin \theta) \\
& -\frac{h^{*}}{4}\left(1-\frac{\pi}{4}\right)\left(\pi b_{\text {fill }}^{*}+\Lambda^{*} \theta\right), \\
\theta= & 2 \arccos \left(1-\frac{b_{\text {fill }}^{*}}{\Lambda^{*}}\right) .
\end{aligned}
$$

The remaining unknown in the preceding equations is $b_{\text {fill }}^{*}$, which is determined by a force balance on the droplet presented in Sec. II.

\section{Necking}

During the necking period additional fluid is being pumped into the droplet as the neck collapses. The increase in size of the droplet during this stage is related to the time that the neck remains open and the flow rate of the dispersed phase. Only a portion of the continuous phase flow collapses the neck and this depends on this correlates with the fraction of the flow that is blocked by the interface [1].

The factor $\beta$ essentially represents the dimensionless necking time $\beta=\Delta t_{\mathrm{sq}}^{*}=\Delta t_{\mathrm{sq}} w_{c}^{2} h / Q_{c}$. To determine $\Delta t_{\mathrm{sq}}^{*}$, van Steijn et al. applied conservation of mass to the continuous phase in order to describe the changing shape of the neck in terms of the radius $R_{n}$ [11]. As the neck collapses the radius becomes larger and the neck approaches the inside corner of the T-junction; once it reaches a critical distance $2 r_{\text {pinch }}$, the rate of collapse increases exponentially. Therefore, the shape of the neck at the point of collapse needs to be known to calculate $\beta$.

Consider the deformable control volume consisting of the continuous phase surrounding the neck as shown in Fig. 5. The change in shape of the control volume during the squeezing process is related to the relative inflow and outflow of the continuous phase across any boundary, which in this case reduces to [13]

$$
\frac{d V_{\mathrm{CV}}}{d t}=Q_{c}\left(1-\frac{A_{\mathrm{bp}}^{*}}{h^{*}}\right) .
$$

The dimensionless bypass area is given as

$$
A_{\mathrm{bp}}^{*}=A_{\text {gap }}^{*}=\left(1-\bar{b}^{*}\right) h^{*}+\left(1-\frac{\pi}{4}\right) \frac{\left(h^{*}\right)^{2}}{2},
$$

where $\bar{b}^{*}$ is the average penetration depth during the necking stage $\bar{b}^{*}=\left(b_{\text {fill }}^{*}+b_{\text {pinch }}^{*}\right)$. Nondimensionalizing Eq. (5) and integrating with respect to time from the initial filling volume 


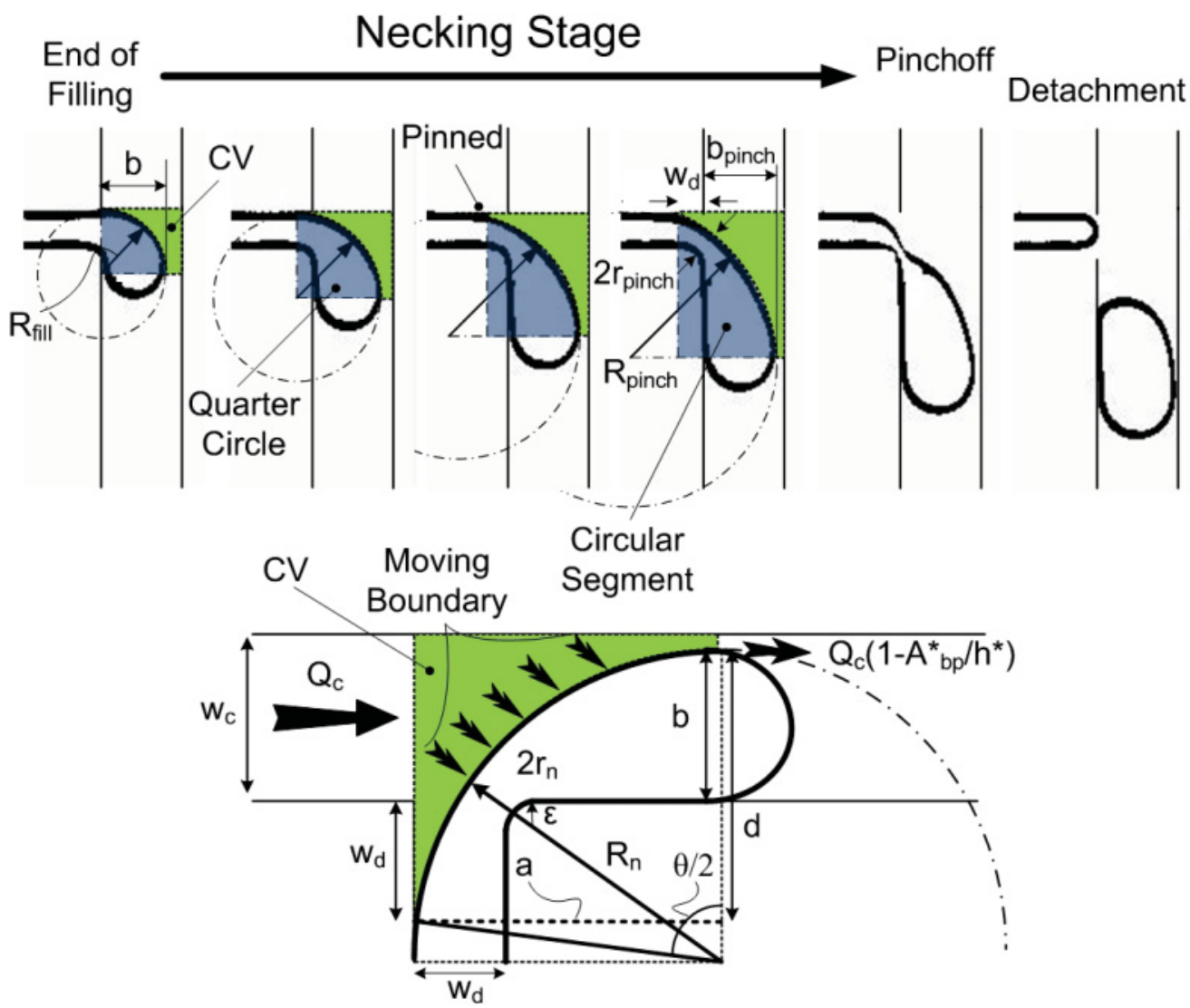

FIG. 5. (Color online) Sequence of traces showing the boundary of the droplet as it evolves during the squeezing stage. At first the neck shape follows a quarter circle until it becomes pinned within the dispersed phase channel at a distance $w_{d}$; it then follows the shape of a circular segment. The control volume around the neck is highlighted in light gray (green). At the bottom, the detailed geometry and flow parameters of the control volume are shown.

$V_{c_{\text {fill }}}^{*}$ to the final pinch volume $V_{c_{\text {pinch }}}^{*}$, the factor $\beta$ can be recovered:

$$
\beta=\left(V_{c_{\text {pinch }}}^{*}-V_{c_{\text {fill }}}^{*}\right)\left(1-\frac{A_{\mathrm{bp}}^{*}}{h^{*}}\right)^{-1}
$$

Here the assumption is made that $1-A_{\mathrm{bp}}^{*} / h^{*}$ represents the fraction of the continuous phase actually squeezing the neck and not bypassing it through the gap [1].

Applying Eq. (2) to the 2D area of the control volume at the end of the filing period we obtain

$$
V_{c_{\text {fill }}^{*}}^{*}=\left(1-b^{*}\right) R_{\text {fill }}^{*}+\left(1-\frac{\pi}{4}\right)\left(R_{\text {fill }}^{*}\right)^{2}+\frac{\pi h^{*}}{4}\left(1-\frac{\pi}{4}\right) R_{\text {fill }}^{*} .
$$

To describe the evolution of neck shape, van Steijn et al. used a quarter circle to approximate the shape of the neck throughout the necking process. However, we found that this approximation consistently underestimated the necking time in the transition regime. At first the neck does in fact follow a quarter-circle shape; however, once the continuous phase penetrates into the side channel a distance $w_{d}$ it remains pinned at this distance while the neck radius continues to grow, resulting in a longer necking period (refer to Fig. 5). At this point, a better description of the neck shape is a half-circular segment [12]. Using the definition of variables in Fig. 5, the back of the droplet is described by

$$
\begin{aligned}
d^{*} & =b_{\text {pinch }}^{*}+\Lambda^{*}, \\
a^{*} & =\sqrt{d^{*}\left(2 R_{\text {pinch }}^{*}-d^{*}\right)} \\
\theta & =2 \arccos \left(1-\frac{b_{\text {pinch }}^{*}+\Lambda^{*}}{R_{\text {pinch }}^{*}}\right), \\
2 r_{\text {pinch }}^{*}-\varepsilon^{*} & =R_{\text {pinch }}^{*}-\sqrt{\left(R_{\text {pinch }}^{*}-b_{\text {pinch }}^{*}\right)^{2}+\left(a^{*}-\Lambda^{*}\right)^{2}},
\end{aligned}
$$

where $R_{\text {pinch }}^{*}$ is calculated for a specific $2 r_{\text {pinch }}^{*}$ by iterating the set of equations above. The final pinch volume for the half-circular segment is then given as

$$
\begin{aligned}
V_{c_{\text {pinch }}}^{*}= & \left(1-b_{\text {pinch }}^{*}\right) a^{*}+\left(1+\Lambda^{*}\right) a^{*} \\
& -\frac{\left(R_{\text {pinch }}^{*}\right)^{2}}{4}(\theta-\sin \theta)+\frac{h^{*} \theta}{4}\left(1-\frac{\pi}{4}\right) R_{\text {pinch }}^{*}
\end{aligned}
$$

Experimental observations showed that for the geometries considered in this study $b_{\text {pinch }}^{*}$ increases by approximately $20 \%$ after the filling stage to the end of necking, capped by the far wall $\left(b_{\text {pinch }}^{*} \leqslant 1\right)$. Therefore, in the following calculations involving the second stage of formation the following expression is used: $b_{\text {pinch }}^{*}=\min \left(1.2 b_{\text {fill }}^{*}, 1\right)$. 


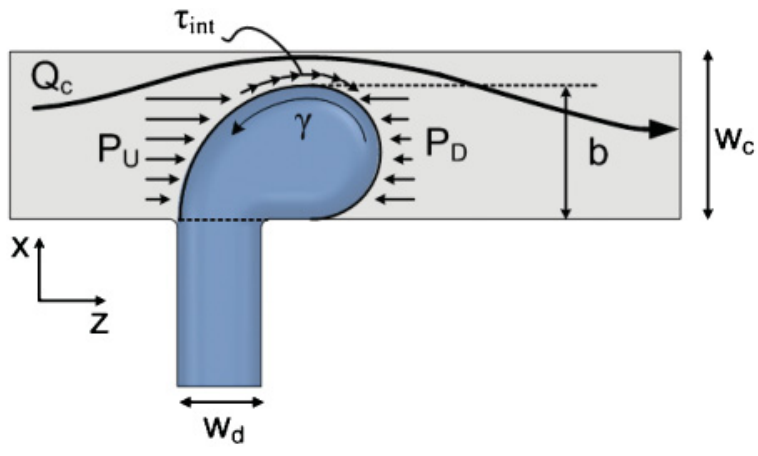

FIG. 6. (Color online) Diagram indicating the forces acting on the emerging droplet in the filling stage. As the droplet grows it obstructs the continuous phase, resulting in a pressure difference across the droplet as well as shear stress on the interface. These two forces are countered by the interfacial tension force, which resists deformation. At some point the droplet reaches a maximum size characterized by the dimension $b$, where the pressure and shear forces exceed the surface tension force and the neck begins to thin.

Once $V_{c_{\text {pinch }}}^{*}$ is known it is substituted into Eq. (6) to determine the necking factor $\beta$. So far the two factors $\alpha$ and $\beta$ have been defined; however, there are still two unknown quantities $b_{\text {fill }}^{*}$ and $2 r_{\text {pinch }}$ that need to be defined.

\section{Force balance on the emerging droplet}

Following the estimates put forth by Garstecki et al. and Christopher et al., three primary forces govern droplet formation: the interfacial tension resisting deformation, the tangential shear acting on the interface, and the squeezing pressure across the droplet (refer to Fig. 6) [3,4]. Necking begins once the stabilizing capillary force is overwhelmed by the shear and pressure forces. The penetration depth $b_{\text {fill }}$ defines this point.

The capillary force is associated with the Laplace pressure difference between the upstream and downstream ends of the emerging droplet interface. Based on the droplet shape at the end of the filling phase (refer to Fig. 4), the upstream interface has a curvature $k_{d}=2 / b+2 / h$, while the downstream curvature is $k_{u}=1 / R_{n}+2 / h$. The sum of these two Laplace pressures, multiplied by the area over which the force is applied, $A \sim b h$, gives the resulting capillary force $F_{\gamma} \approx-\gamma h$. The viscous shear force acting on the droplet may be estimated as the average shear stress on the interface multiplied by the exposed area $\mathrm{A} \sim b h$ and is given as $F_{\tau} \approx$ $\tau_{\text {int }} b h$. The partial blockage of the continuous phase flow by the emerging interface creates a pressure drop across the droplet. The resultant force is the difference between the upstream and downstream pressures multiplied by the exposed area $A \sim b h$ : $F_{p} \approx \Delta \mathrm{P}_{u-d} b h$. The filling stage ends when the three forces sum to zero:

$$
F_{\tau}+F_{P}+F_{\gamma}=0 .
$$

Initial models by Garstecki et al. and Christopher et al. estimated the pressure drop through a simple scaling analysis based on the Hagen-Poiseuille relation in a thin gap $[3,4]$. Here the same approach is taken to quickly estimate the relative magnitude of the shear and pressure forces. Approximating the shear rate on the droplets as the average velocity through the gap $w_{\text {gap }}=\left(w_{c}-b_{\text {fill }}\right)$ divided by the height $\tau_{\text {int }} \approx \mu_{c} u_{\text {gap }} / h$, the resulting shear force becomes

$$
F_{\tau} \approx \frac{\mu_{c} b_{\text {fill }}}{w_{\text {gap }} h} Q_{c} .
$$

The pressure drop can be approximated using the lubrication analysis for pressure drop between two parallel plates $\Delta P_{u-d} \approx 12 Q_{c} \mu_{c} b_{\text {fill }} /\left(w_{\text {gap }} h\right)^{2}$ resulting in a pressure force give by [13]

$$
F_{P} \approx \frac{12 \mu_{c} b_{\text {fill }}^{2}}{w_{\text {gap }}^{2} h} Q_{c} .
$$

Taking the ratio of the two forces in order to estimate their importance we obtain

$$
\frac{F_{\tau}}{F_{P}} \approx \frac{w_{\text {gap }}}{12 b_{\text {fill }}}=\frac{\left(w_{c}-b_{\text {fill }}\right)}{12 b_{\text {fill }}} .
$$

Under typical conditions for droplet generation in the Tjunction $\left(\mathrm{Ca}=0.0001-0.02\right.$ and $\left.b^{*}=0.6-1\right)$, the shear stress force is only $0-5 \%$ of the pressure force. Therefore, it is safe to neglect the contribution of shear stress from the force balance on the emerging interface.

To accurately predict $b_{\text {fill }}$ the remaining issue is to develop a model that captures the pressure drop across the droplet correctly. This is not straightforward as the flow profile through the gap is quite complex since the region is comprised of three walls obeying the no-slip conditions and the fourth is the interface where the shear and velocity between the two phases must be continuous. In addition, the shape of the interface creates a curved boundary in both the out-of-plane and in-plane orientations, resulting in a 3D flow profile throughout the gap, which does not lend itself to a simple analytical solution. For this reason, a semianalytical solution was developed based on asymptotic limits to the flow profile using the assistance of numerical simulations.

\section{PRESSURE DROP CALCULATION}

Tchikanda et al. numerically modeled pressure and shear driven flow in open rectangular microchannels with one boundary being a curved interface. Their intended application was the design of evaporative microfluidic cooling devices with parallel liquid-vapor flows [14]. The authors performed 2D numerical simulations to obtain the flow field for various gap shapes and then developed analytical solutions by blending asymptotic results for limits of channel aspect ratio and interface curvature. For example, when the gap is large and the interface is flat, the flow resembles that between two parallel plates. If the interface is curved and the gap is small then flow is primarily through the two disjoint corners (see Fig. 7).

Tchikanda et al. provide separate solutions for shear and pressure driven flow that may be combined due to the linearity of the Navier-Stokes equations in the lubrication limit [14]. The analytical solutions are easy to apply and are accurate to within a few percent compared to the numerical solutions. 

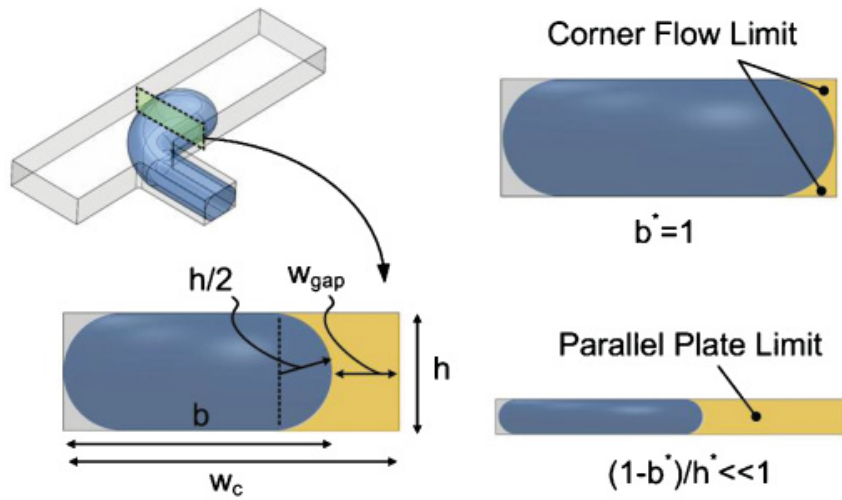

FIG. 7. (Color online) Geometry of the gap where the fluid bypasses the droplet for flow fluid flow model of Tchikanda et al. Examples of the limits of corner flow and parallel plate flow are also illustrated.

The authors provide solutions in terms of the dimensionless average velocity in the area occupied by the fluid:

$$
\bar{u}^{*}=\frac{Q_{c} \mu_{c}}{A h^{2}(-\partial P / \partial z)}
$$

where $h$ is the height of the channel, $A$ is the area of the conduit, $\mu$ is the viscosity, $Q$ is the flow rate, and $-\partial P / \partial z$ is the pressure gradient. Integrating the pressure gradient over a uniform conduit of length $L$, the equation can be rearranged into a format similar to the Hagen-Poiseuille law [13]:

$$
\frac{\Delta P}{Q}=R_{\mathrm{hyd}}=\frac{L}{\bar{u}^{*} A h^{2}} \mu,
$$

where $R_{\text {hyd }}$ is the hydrodynamic resistance and $L / \bar{u}^{*} A h^{2}$ is the geometric component.

Using the notation of Tchikanda et al., the geometry of the gap corresponds to an interface with a contact angle of $0^{\circ}[14]$. The relevant dimensions for the gap geometry are shown in Fig. 7. The nondimensional area is then given as

$$
A_{\text {gap }}^{*}=\left(1-b^{*}\right) h^{*}+\frac{1}{2}\left(h^{*}\right)^{2}\left(1-\frac{\pi}{4}\right) \text {. }
$$

The average dimensionless velocity is provided as a blend of asymptotic solutions $u_{1}^{*}$ and $u_{2}^{*}$, given by the following expression:

$$
\bar{u}^{*}=\left[\frac{\left(u_{1}^{*} u_{2}^{*}\right)^{m_{1}}}{\left(u_{1}^{*}\right)^{m_{1}}+\left(u_{2}^{*}\right)^{m_{1}}}\right]^{1 / m_{1}} .
$$

The expression approaches $u_{1}^{*}$ in the limit of $u_{1}^{*} \gg u_{2}^{*}$ and $u_{2}^{*}$ in the opposite limit of $u_{1}^{*} \ll u_{2}^{*}$. The shape of the blend is controlled by the fitting parameter $m_{1}$. The first asymptote $u_{1}^{*}$ corresponds to a wide aspect ratio gap $\lambda_{\text {gap }}=h^{*} /\left(1-b^{*}\right)$ or flow between two infinite plates (top and bottom of the microchannel) $u_{1}^{*}=1 / 12$. The second asymptote approaches two limits. The first is when the gap is very shallow and the interface is flat; the second is when the crest of the meniscus is in contact with the wall and flow is only through the corners:

$$
u_{2}^{*}=\left[\left(\frac{1}{a \lambda_{\text {gap }}+3 \lambda_{\text {gap }}^{2}}\right)^{m_{2}}+\left(u_{\mathrm{co}}^{*}\right)^{m_{2}}\right]^{1 / m_{2}},
$$

where $u_{\mathrm{co}}^{*}=0.0027$ is the corner asymptotic limit.

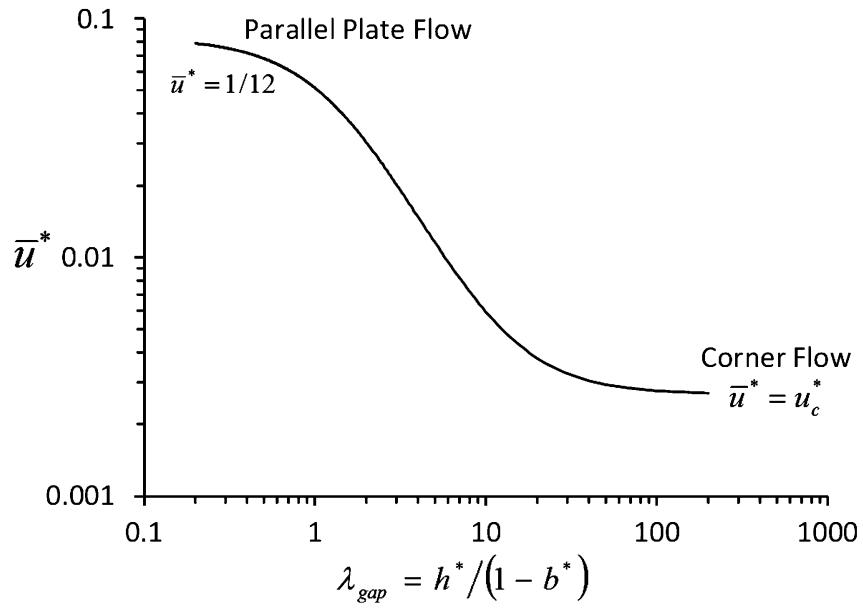

FIG. 8. Dimensionless mean velocity as a function of the gap aspect ratio with the two limits of parallel plate flow and corner flow identified.

We repeated the numerical simulations and found different optimum fitting parameters $m_{1}=1.25, m_{2}=0.90, m_{3}=1.88$, and $a=7.90$ for our geometry. With this curve fit the average error compared to the numerical results is less than $1.5 \%$. The shape of the average mean velocity function including the two asymptotes is shown in Fig. 8.

Two additional effects are absent in the approximation by Tchinkada et al. that are present in the situation of the bypassing flow. First, the solutions correspond to a conduit with a uniform cross section. Curvature of the droplet in the $x-z$ plane creates a nonuniform gap profile. Second, the analysis assumes a zero-shear boundary at the interface, which is appropriate for conditions where the continuous phase viscosity is much greater than the dispersed phase viscosity $(\eta \rightarrow 0)$. Higher viscosity ratios act to modify the shear stress condition on the interface, resulting in an increase in resistance to flow through the gap. We performed numerical simulations and developed additional factors $L_{\text {eff }}$ for the curvature and $g\left(\eta, \lambda_{\text {gap }}\right)$ for the viscosity contrast that modify Eq. (16).

\section{A. Effective pressure drop length}

The interface curvature in the $x-z$ plane creates a constantly varying gap profile along the length of the droplet. The pressure drop generally scales with the cube root of the smallest dimension; therefore, one expects that the effective length over which the majority of the pressure drop occurs in the gap will vary with the penetration depth $b^{*}$. Such a dependence was derived by Stone in his study of lubrication flow through shallow curved gaps [15]. Because of the complex shape of the droplet, a solution to the pressure drop requires a full $3 \mathrm{D}$ analysis of the flow field. For this reason a numerical study of the flow field through the gap was performed using COMSOL Multiphysics 4.1 .

Simulations were performed for different droplet sizes $b^{*}=0.5-1$ and channel heights $h^{*}=0.2,0.35$, and 0.5. Only one half of the droplet was simulated due to symmetry along the $x-z$ plane as shown in Fig. 9. Five channel lengths were added before and after the droplet to ensure that the flow field is properly realigned at the entrance and exit. 


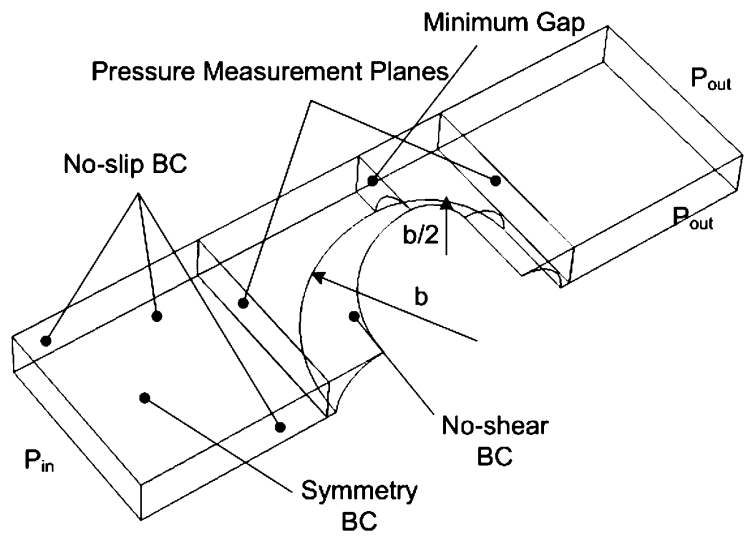

FIG. 9. Simulation domain of the 3D numerical model. Calculations were performed only for the continuous phase. Boundary conditions are no slip at all the channel walls and no shear at the droplet interface.

No-slip conditions were applied to all microchannel walls and a no-shear condition was applied to the interface of the droplet. A symmetry boundary condition (no shear) was applied at the plane of symmetry. At the entrance and exit pressures were fixed. For each simulation the pressure drop was measured at two planes just before and after the droplet (the total distance between planes is $1.5 b$ ). Additionally, the average velocity in the channel was measured to calculate the total flow rate and determine $\bar{u}^{*}$.

The goal here is to still utilize the curve fits provided by Tchiknada et al. because of their inherent simplicity and accuracy while adding the influence of the out-of-plane curvature. This is accomplished by calculating the equivalent length of channel of the uniform cross section for the minimum gap, which will produce the same pressure drop as the 3D numerical simulation. Mathematically this relationship is given as the ratio of the pressure drop from the 3D numerical simulations to the pressure drop from the asymptotic model $\left(L_{\text {eff }}^{*}=L_{\text {eff }} / w_{c}=1.5 b^{*} \Delta P_{3 \mathrm{D}} / \Delta P_{\text {asy }}\right)$. Figure 10 plots the ratio $\Delta P_{3 \mathrm{D}} / \Delta P_{\text {asy }}$ as a function of $b^{*}$ for a range of $h^{*}$ for droplets following the shape of $w_{d} \leqslant b_{\text {fill }}$. Normalizing the 3D result by the asymptotic model causes all of the data to collapse onto

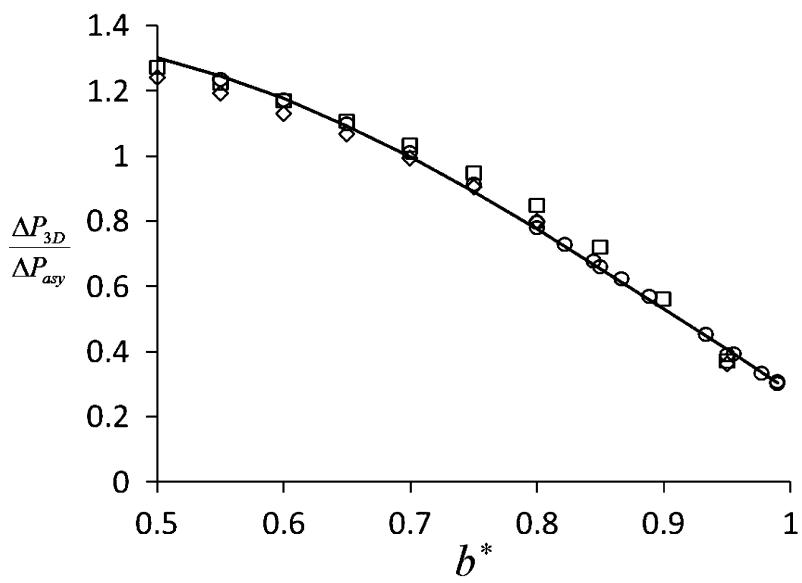

FIG. 10. Effective pressure drop length $L_{\mathrm{eff}}^{*}$. Data correspond to $h^{*}=0.2(\diamond), 0.35(\square)$, and $0.5(\circ)$. a single curve. The relationship for $L_{\text {eff }}^{*}$ can be described by a blend of limiting solutions consisting of a linear relationship $L_{1}^{*}=-2.66 b^{*}+2.88$ and an asymptote $L_{2}^{*}$ :

$$
L_{\text {eff }}^{*}=L_{\text {drop }}^{*}\left[\frac{\left(L_{1}^{*} L_{2}^{*}\right)^{k}}{\left(L_{1}^{*}\right)^{k}+\left(L_{2}^{*}\right)^{k}}\right]^{1 / k} .
$$

For the case $w_{d} \leqslant b_{\text {fill }}$ the coefficients are $L_{2}^{*}=1.3, k=6$, and $L_{\text {drop }}^{*}=1.5 b_{\text {fill }}^{*}$; for $w_{d}>b_{\text {fill }}, L_{2}^{*}=1, k=3$, and $L_{\text {drop }}^{*}=$ $\Lambda^{*}+b_{\text {fill }}^{*}$.

\section{B. Viscosity contrast effect}

Simulations were performed in two dimensions emulating the cross-sectional view of the microchannel at the minimum gap point. A pressure gradient was applied in the continuous phase while no pressure gradient was applied in the portion of the dispersed phase. Thus flow in the dispersed phase is caused by the drag at the interface created by the bypassing continuous flow. This situation approximates the actual conditions prevalent in the gap during droplet formation. Generally, $Q_{c}>$ $Q_{d}$ and the continuous phase must flow through a smaller area $\sim\left(1-b^{*}\right)$ then the dispersed phase $\sim b^{*}$. Thus the average velocity in the gap region is typically an order of magnitude higher than the velocity within the droplet, so that as an approximation, one can consider the flow in the drop to be stagnant in comparison. For the boundary conditions, no slip is applied along all the microchannel walls and continuity at the interface boundary.

Simulations were performed for gaps varying between $b^{*}=0.6$ and 1 and viscosity contrasts between $\eta=1 / 100$ and 1 . Figure 11 plots the relative change in resistance to flow as compared to the no-shear case for different viscosity contrasts. The trend is a nonmonotonic function that generally increases as the gap closes $\left(b^{*}=0.5-1\right)$. Peculiarly, a small decrease in the relative pressure drop occurs around $b^{*}=0.95$ presumably due to the unique geometry and competing boundary conditions on the flow within the gap region. A two-step fit is applied to the function approximating the first part $\lambda_{\text {gap }}<2$ with a linear curve fit $g\left(\eta, \lambda_{\text {gap }}\right)=a(\eta) \lambda_{\text {gap }}+1$

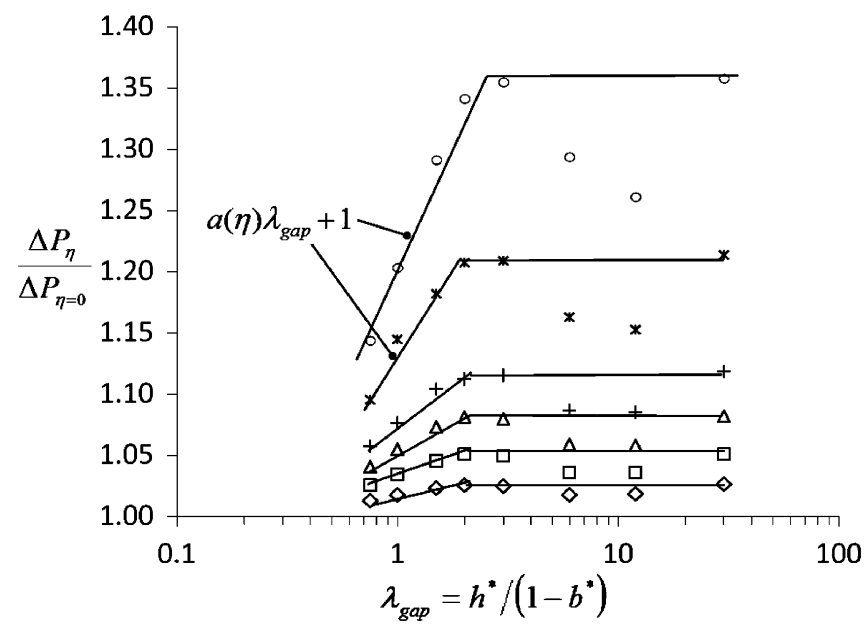

FIG. 11. Effect of viscosity contrast on the pressure drop from the $2 \mathrm{D}$ numerical simulations. Data correspond to $\eta=0.1(\diamond)$, $0.2(\square), 0.33(\Delta), 0.5(+)$, and $\eta=1(*)$. 
and the second $\lambda_{\text {gap }} \geqslant 2$ as a constant. The slope of the linear function scales proportionally with the viscosity contrast and has the form $a(\eta)=0.1 \eta\left(R^{2}=0.96\right)$. The piecewise function that defines the effect of the viscosity contrast is thus given by

$$
g\left(\eta, \lambda_{\text {gap }}\right)= \begin{cases}0.1 \eta \lambda_{\text {gap }}+1, & \lambda_{\text {gap }}<2 \\ 0.2 \eta+1, & \lambda_{\text {gap }} \geqslant 2 .\end{cases}
$$

This relationship is used to modify the pressure drop calculation provided by Tchinkada et al. and account for the effect of viscosity contrast.

\section{Reconstituted force balance}

By integrating Eq. (16) the pressure difference over the droplet can be calculated as

$$
\Delta P_{u-d}=\frac{L_{\text {eff }} Q_{c_{\text {gap }}} \mu_{c}}{h^{2}\left(A_{\text {gap }} \bar{u}_{\text {gap }}^{*}\right)} g\left(\eta, \lambda_{\text {gap }}\right) .
$$

Substituting into Eq. (11) and neglecting the shear stress contribution, the force balance in dimensionless terms becomes

$$
\frac{h^{*}}{\mathrm{Ca}}=\frac{1}{2} \frac{L_{\mathrm{eff}}^{*} g\left(\eta, \lambda_{\mathrm{gap}}\right)}{\left(A_{\mathrm{gap}}^{*} \bar{u}_{\mathrm{gap}}^{*}\right)} \frac{b_{\mathrm{fill}}^{*}}{\left(2-b_{\mathrm{fill}}^{*} / R_{n}^{*}\right)} .
$$

For the case $w_{d} \leqslant b_{\text {fill }}, R_{n}=b_{\text {fill }}$; for $w_{d}>b_{\text {fill }}, R_{n}=w_{d}$ when calculating the force balance. This shows that the controlling parameter for the fill volume is $h^{*} / \mathrm{Ca}$. Equation (23) is iterated until a convergent solution for $b_{\text {fill }}^{*}$ is found. Once $b_{\text {fill }}^{*}$ is known it is substituted back into Eq. (4a) or (4b) to calculate $\alpha_{\text {fill }}$. A comparison with experiment indicates that a prefactor of $C=$ $1 / 2$ fits well-a peculiar value as it suggests that the surface tension force is underestimated by a factor of 2 or the pressure force is overestimated by a factor of 2 . The reason for this is not quite known, but there are several explanations that can be suggested. First, the force balance in the streamwise direction is not the only contribution to the deformation of the emerging interface as a force in the cross-stream direction also exists. This force is caused by the pressure difference within the droplet and the average pressure in the gap $F_{p x} \propto \Delta P_{u-d} / 2$, where the factor $1 / 2$ appears. A second explanation is that the pressure drop is reduced because the continuous phase is also able to bypass the emerging interface through additional conduits such as the top and bottom films or the near channel wall. Third, the surface tension force may be underestimated as we assumed that the out-of-plane curvature $\sim 2 / h$ remained the same for the front and back of the droplet, resulting in the cancellation of out-of-plane curvature effects. It is well known that the curvature of the front cap increases relative to the back cap when droplets travel in microchannels [16]. If we presume that this effect also occurs during drop formation then it would contribute to an increase in the surface tension force. Most likely a combination of these effects produce the prefactor of $1 / 2$ and untangling them would require a detailed numerical study, which is beyond the scope of this current work. Nevertheless, the penetration depth is well described by Eq. (23) when a prefactor of $1 / 2$ is used, as will be shown later on.

From the force balance a criterion can also be found for the point where the system changes from purely squeezing into the transition regime. Considering the limit for the squeezing regime to be when the interface reaches the far wall during the first stage, Eq. (23) may be rearranged to determine the critical $\mathrm{Ca}$ for the squeezing regime:

$$
\mathrm{Ca}_{\mathrm{sq}} \leqslant 1.7 e^{-3} \frac{\left(h^{*}\right)^{3}}{g\left(\eta, \lambda_{\text {gap }}\right)} .
$$

This result shows that the critical limit for squeezing is very sensitive to the height of the microchannel. Furthermore, squeezing occurs for very low $\mathrm{Ca}_{\mathrm{sq}}=2.05 e^{-4}$ values $\left(h^{*}=0.5\right.$ and $\eta=0$ ), suggesting that, in practice, T-junction generators always operate in the transition regime to some degree.

\section{CALCULATION OF THE PINCHOFF POINT}

In order to incorporate the observed $\mathrm{Ca}$ and viscosity dependence on $2 r_{\text {pinch }}^{*}$ an alternative approach was taken. The idea is that pinchoff occurs when the Laplace pressure difference between the front and back of the droplet generates a backward flow that exceeds some multiple $m$ of the bypassing flow $Q_{c} A_{\mathrm{bp}}^{*} / h^{*}$. The Laplace pressure driven fluid flow around the drop may be approximated by

$$
\Delta P_{\mathrm{LP}}=\gamma\left(\frac{1}{R_{n}}+\frac{1}{r_{n}}\right)-\gamma\left(\frac{2}{b_{\text {pinch }}}+\frac{2}{h}\right),
$$

where the pressure created by the bypassing flow is given as

$$
\Delta P_{\mathrm{LP}}=\frac{L_{\mathrm{eff}} \mu_{c} g\left(\eta, \lambda_{\mathrm{gap}}\right)}{h^{2}\left(A_{\mathrm{gap}} \bar{u}_{\mathrm{gap}}^{*}\right)} m Q_{c} \frac{A_{\mathrm{bp}}^{*}}{h^{*}} .
$$

Ignoring the smaller term of $1 / R_{n}$ and rearranging Eqs. (25) and (26), the critical neck thickness is given as

$$
2 r_{\text {pinch }}^{*}=\frac{h^{*}}{1+\frac{h^{*}}{b_{\text {pinch }}^{*}}+\frac{m}{2} \frac{\mathrm{Ca}}{h^{*}} \frac{L_{\text {eff }}^{*} g\left(\eta, \lambda_{\text {gap }}\right)}{\bar{u}_{\text {gap }}^{*}}} .
$$

In the case of $\mathrm{Ca} \rightarrow 0$, then $b^{*} \rightarrow 1$ and the limit from van Steijn et al. is recovered for the squeezing regime [10]. Comparisons with experiments show that the optimum fitting parameter is $m=1$. This means that the sudden collapse of the neck occurs when the back pressure matches the hydrodynamic pressure from the bypassing flow. Overall the alternative model for the neck is more robust since it includes the interfacial tension and viscosity in $\mathrm{Ca}$ as well as the resistance to fluid flow through the gutters around the drop through $\bar{u}^{*}$.

\section{OPERATIONAL PARAMTERS}

In this section the other important operational parameters are calculated, including the volume of oil injected between the droplets, droplet spacing, and the frequency of formation. The total volume of oil injected can be broken down into three contributions: (a) oil that bypasses the droplet during the lag stage when the interface is inside the dispersed channel, (b) oil that bypasses the emerging interface during the drop filling stage, and (c) oil that continues to bypass the droplet during the squeezing stage:

$$
V_{c}=V_{c_{\mathrm{lag}}}+V_{c_{\text {fill }}}+V_{c_{\text {neck }}} \text {. }
$$




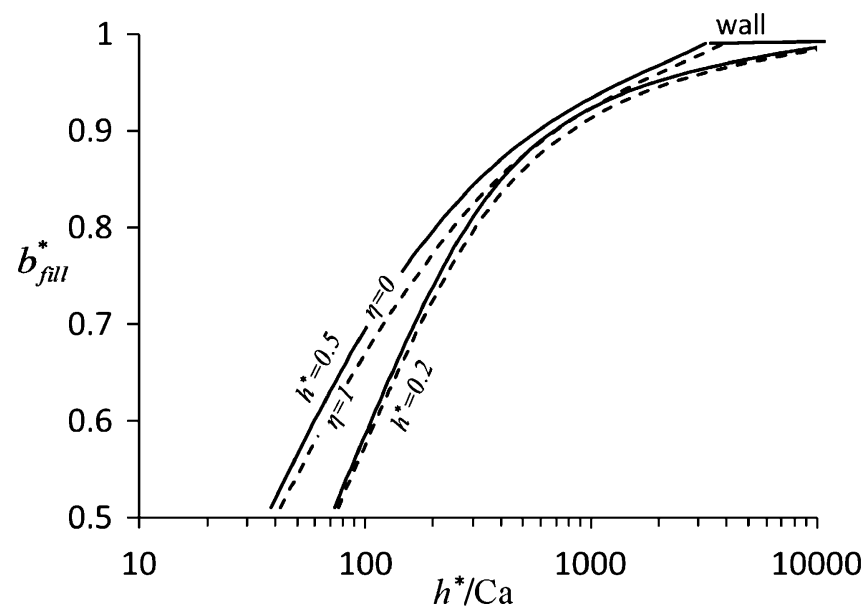

FIG. 12. Plot of $b_{\text {fill }}^{*}$ with respect to $h^{*} / \mathrm{Ca}$ for two different channel aspect ratios and viscosity ratios.

The volume is related to the length of each stage and the oil flow rate, which is assumed to remain constant throughout the three stages:

$$
V_{c}=\Delta t_{\text {lag }} Q_{c}+\Delta t_{\text {fill }} Q_{c}+\Delta t_{\text {neck }} Q_{c} .
$$

Note that during the necking stage $Q_{c}$ is used because all of the flow either collapses the neck or bypasses the droplet and thus eventually contributes to the spacing. The length of each stage is given as $\Delta t_{\text {lag }}=\alpha_{\text {lag }} w_{c}^{2} h / Q_{d}, \Delta t_{\text {fill }}=\alpha_{\text {fill }} w_{c}^{2} h / Q_{d}$, and $\Delta t_{\text {neck }}=\beta w_{c}^{2} h / Q_{c}$. The nondimensional form becomes

$$
V_{c}^{*}=\frac{\alpha_{\text {lag }}+\alpha_{\text {fill }}}{\phi}+\beta
$$

The rate of production and droplet spacing are additional parameters that are important in the design of a droplet generator and can be derived using arguments similar to those that were employed in the calculation of the droplet volume.

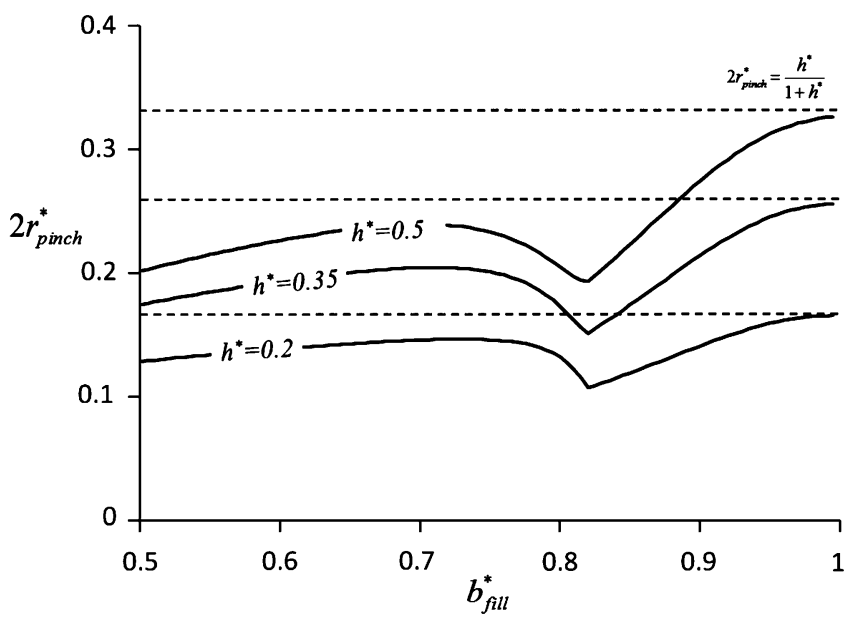

FIG. 13. Calculations of the new critical neck thickness for $h^{*}=$ $0.2,0.35$, and 0.5 .

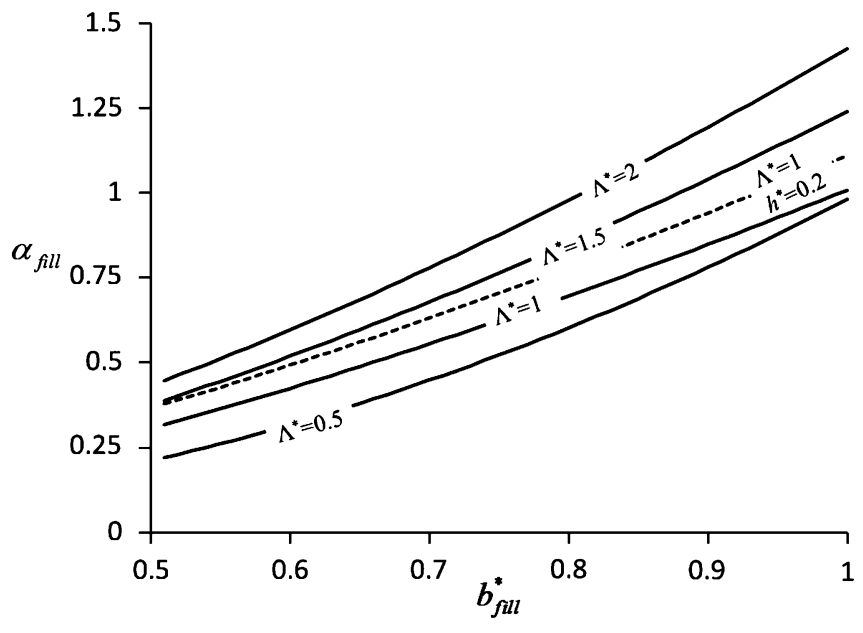

FIG. 14. Plot of $\alpha$ with respect to $b_{\text {fill }}^{*}$ for various T-junction geometries with a base value of $h^{*}=0.5$ and $\varepsilon^{*}=0$.

The spacing $\lambda=L_{\text {drop }}+L_{c}$ may be approximated by assuming $V^{*} \sim L^{*}$.

$$
\lambda^{*}=\left(\alpha_{\text {lag }}+\alpha_{\text {fill }}\right)\left(\frac{\phi+1}{\phi}\right)+\beta(\phi+1) .
$$

The total time for droplet production is the sum of the three stages: $t_{\text {drop }}=\Delta t_{\text {lag }}+\Delta t_{\text {fill }}+\Delta t_{\text {sq }}$. The period over which a droplet forms is

$$
t_{\mathrm{drop}}^{*}=\frac{\alpha_{\mathrm{lag}}+\alpha_{\mathrm{fill}}}{\phi}+\beta
$$

The normalized frequency is then the reciprocal of this equation:

$$
f^{*}=\frac{\phi}{\alpha_{\text {lag }}+\alpha_{\text {fill }}+\phi \beta} .
$$

For the reader's benefit, the complete set of equations is concisely presented in Ref. [17].

\section{MODEL SUMMARY}

Plots for the parameters $b_{\text {fill }}^{*}, 2 r_{\text {pinch }}^{*}, \alpha_{\text {fill }}$, and $\beta$ for various conditions and T-junction designs are provided Figs. 12-15.

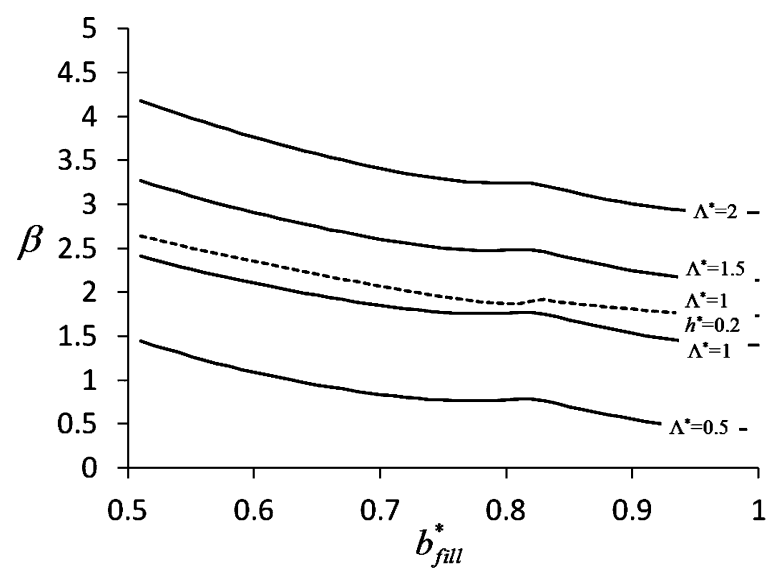

FIG. 15. Plot of $\beta$ with respect to $b_{\text {fill }}^{*}$ for various T-junction geometries with a base value of $h^{*}=0.5$ and $\varepsilon^{*}=0$. 


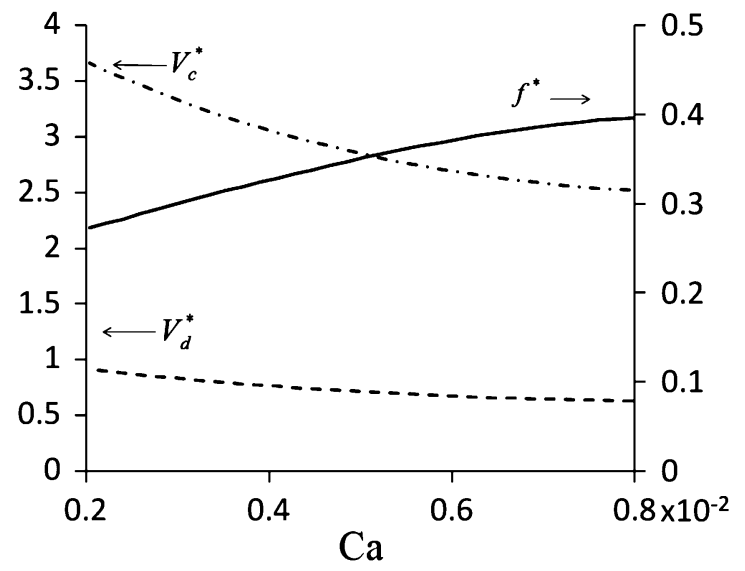

FIG. 16. Variation droplet generation for a T-junction geometry of $h^{*}=0.5$ and $\Lambda^{*}=0.5$ for a fixed flow rate $\varphi=0.25$ against the capillary number.

The most important parameters governing droplet formation are the T-junction geometry $\left(w_{c}, \mathrm{w}_{d} h\right)$ and the term $h^{*} / \mathrm{Ca}$ as it governs $b_{\text {fill }}^{*}$ and $2 r_{\text {pinch }}^{*}$.

Consider Fig. 12, which shows the calculation for $b_{\text {fill }}^{*}$ for different $h^{*}$ and two different viscosity ratios $\eta=0$ and 1 . The plot shows that the relationship between $b_{\text {fill }}^{*}$ and $h^{*} / \mathrm{Ca}$ is nonlinear and as well as a modest dependence on $h^{*}$ and a weak dependence on viscosity contrast. Overall, $b_{\text {fill }}^{*}$ increases with $h^{*} / \mathrm{Ca}$ until it becomes capped at the wall $b_{\text {fill }}^{*}=1$. Often this critical point is associated with the transition into the purely squeezing regime of droplet formation, as predicted by Eq. (24).

Figure 13 plots the ratio of critical neck thickness against $b_{\text {fill }}^{*}$. The profile is nonlinear with an inflection point at $b_{\text {fill }}^{*}=$ 0.833 , which is caused by the relation $b_{\text {pinch }}^{*}=1.2 b_{\text {fill }}^{*}$ and the limit $b_{\text {pinch }}^{*} \leqslant 1$. To the left of the inflection point $b_{\text {fill }}^{*}$ is still free and the critical neck thickness increases; however, it then decreases towards the inflection point because the gap closes, thus increasing the resistance to flow around the droplet. To the right of the inflection point $b_{\text {pinch }}^{*}$ is capped at $b_{\text {pinch }}^{*}=1$ and all of the variables in Eq. (27) are constant, except for the Ca term, which continues to decrease so that Eq. (27) approaches the limit $2 r_{\text {pinch }}^{*} \rightarrow h^{*} /\left(1+h^{*}\right)$.

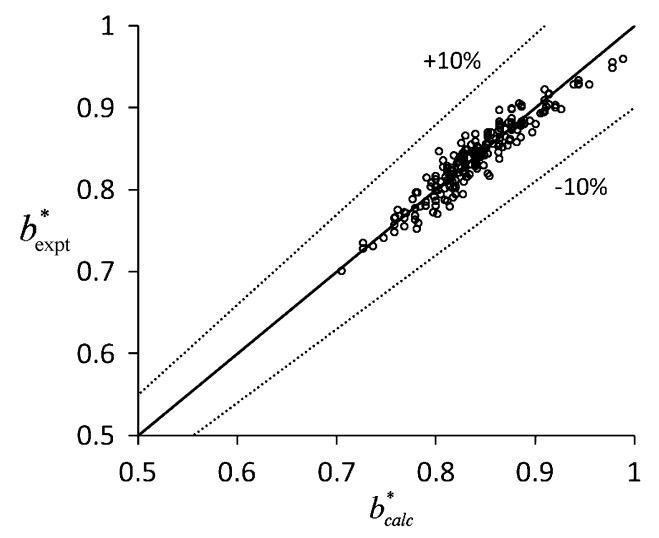

FIG. 17. Parity plot of $b_{\text {fill }}^{*}$ for all experiments. The solid line is perfect parity and the dashed lines are $\pm 10 \%$.

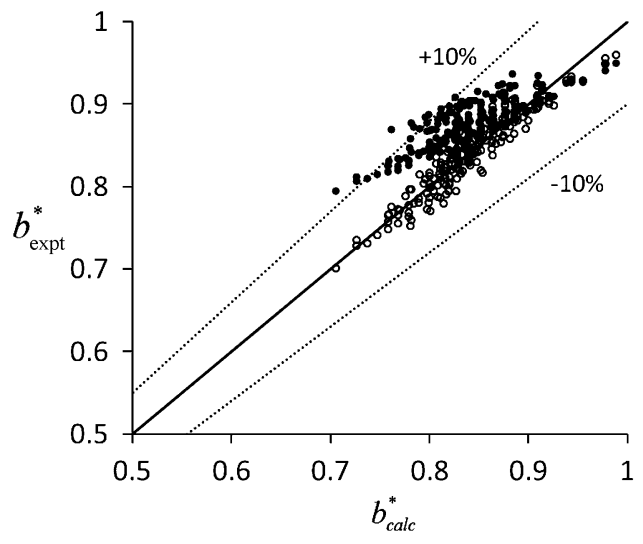

FIG. 18. Parity plot of $b_{\text {fill }}^{*}$ for experiments with $L_{\text {eff }}^{*}$ and $g\left(\eta, \lambda_{\text {gap }}\right)$ : silicone oil (०) and with a constant $L_{\text {eff }}^{*}=0.25(\bullet)$. The solid line represents the perfect parity and the dashed lines are $\pm 10 \%$.

Both $\alpha_{\text {fill }}$ and $\beta$ are primarily governed by the geometry of the T-junction generator, with the width ratio $\Lambda^{*}$ having a stronger influence than the height-to-width ratio $h^{*}$, as shown in Figs. 14 and 15. As expected, the fill volume $\alpha_{\text {fill }}$ decreases with $b_{\text {fill }}^{*}$, which corresponds to higher $\mathrm{Ca}$. What may be a surprise is that the dimensionless necking time $\beta$ actually increases with higher $\mathrm{Ca}$ (seen in the plot as decreasing $b_{\text {fill }}^{*}$ ). There are two contributions to this effect: (i) the emerging interface blocks less of the crossflow, so a smaller fraction of the continuous phase is directed to collapsing the neck and (ii) higher $\mathrm{Ca}$ results in a lower $2 r_{\text {pinch }}^{*}$ value, which means the neck is open longer.

To illustrate the changing performance of a T-junction generator with higher operational speeds (i.e., higher $\mathrm{Ca}$ ), the variation in $V_{d}^{*}, V_{c}^{*}$, and $f^{*}$ is plotted in Fig. 16 for a standard generator design $\left(\Lambda^{*}=0.5\right.$ and $\left.h^{*}=0.3\right)$ while keeping the flow ratio constant $\varphi=0.3$. At lower speeds $(\mathrm{Ca} \rightarrow 0)$ droplets are larger and spaced farther apart, which also means that they are produced at a comparatively lower rate. As $\mathrm{Ca}$ increases smaller droplets are formed closer together and the rate of production increases accordingly. This example clearly shows the complex operational behavior of a T-junction generator within the squeezing-to transitional regimes.

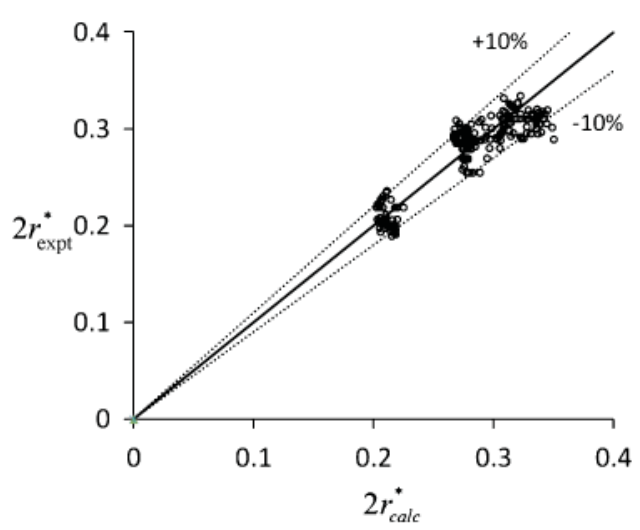

FIG. 19. (Color online) Parity plot of $2 r_{\text {pinch }}^{*}$ for all experimental measurements. 


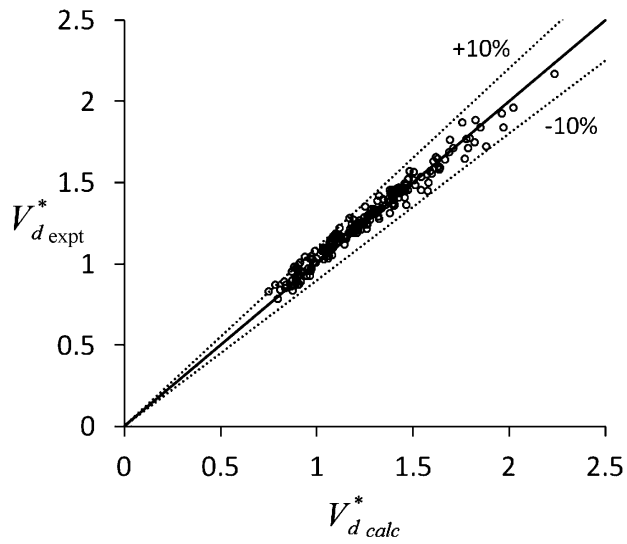

FIG. 20. Parity plot of the droplet volume for all experimental measurements.

\section{MODEL VALIDATION}

The theoretical model was validated by comparing it to experimental data for T-junctions with $\Lambda^{*}=0.34-1$ and $h^{*}=0.3-0.6$ under conditions ranging from $\eta=0.12$ to 1.7 and $\mathrm{Ca}=0.001$ to 0.008 . Details on the experimental procedures were presented in the preceding paper [1]. This set of data covers a wide range of conditions that are typically associated with the transition between squeezing and dripping $\left(b_{\text {fill }}^{*}=\right.$ $0.7-1)$. All data that are presented were first screened to verify that the flow rates are constant during the formation process. This was accomplished by the tracking droplet velocity $u_{d}$ as it is directly proportional to the total flow rate $Q_{c}+Q_{d}$ and by monitoring oscillations in droplet size $V_{d}$ and spacing $\lambda$ as these two metrics are sensitive to the relative flow rate $\varphi$. During the screening process we included only data with $<5 \%$ variance in all three metrics. Therefore, we are confident that the condition of relatively constant flow is satisfied.

The effectiveness of the model is measured by a series of parity plots that compare the predicted penetration depth, droplet volume, oil volume, and production frequency produced by the model to that measured in the experiments. Perfect parity exists when the data fall along the solid black line in the following figures.

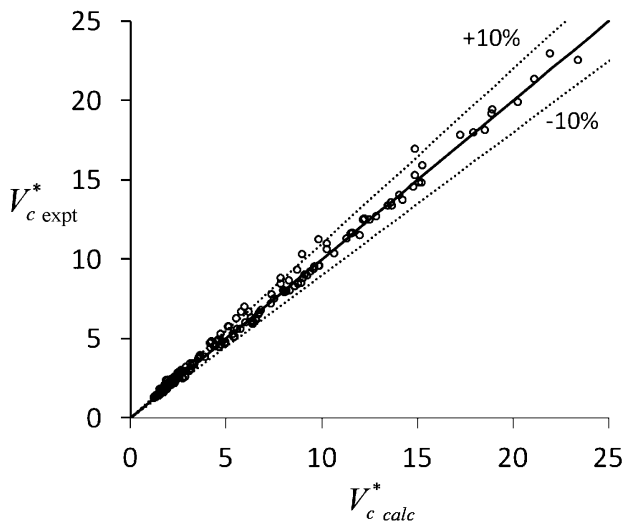

FIG. 21. Parity plot of the continuous phase volume for all experimental data points.

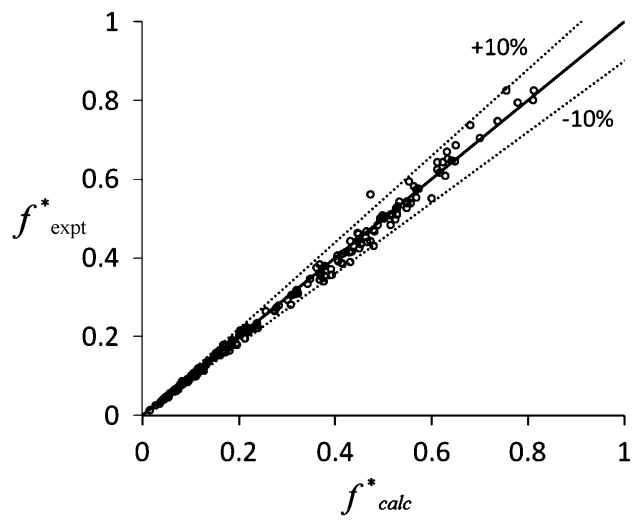

FIG. 22. Parity plot for the generation frequency for all experimental data.

Figure 17 presents the overall accuracy for the calculation $b_{\text {fill }}^{*}$ from the new force balance for all the experiments. Generally, the predictions are very good as most of the data fall within an error range of $\pm 10 \%$, which is deemed acceptable given the potential errors involved in measuring all the parameters from the videos. Figure 18 demonstrates the importance of including $L_{\mathrm{eff}}^{*}$ and $g\left(\eta, \lambda_{\mathrm{gap}}\right)$ in the force balance. One could see that the omission of these two effects results in a systematic overestimation of the penetration depth.

Figure 19 presents a comparison between predicted and measured pinchoff. Good agreement is found, although there is some scatter due to the difficulties measuring the exact moment of collapse. Predictions for $V_{d}^{*}, V_{c}^{*}$, and $f^{*}$ are provided in Figs. 20-22, with an excellent correlation between the experimental and model results as all data fall within $\pm 10 \%$ of parity. This suggests that the model developed herein is successful in predicting the performance of the T-junction generator in the squeezing-to-transition regime without excessive use of correlations. These results verify that the alternative model is able to accurately predict the performance of the T-junction generator in the squeezing-totransition regime.

\section{CONCLUSION}

In this paper II of our two-part series [1], we presented a physical model for the formation of droplets in a microfluidic T-junction generator operating in the squeezing-to-transition regime. The model consists of three parts. The first is a geometric description of the droplet shape and neck during the formation process in the transition regime. This was followed by an alternative force balance to calculate the initial fill volume that includes hydrodynamic resistance of the gap by incorporating the analytical approximations of Tchikanda $e t a l$. [14]. Additional modifications to the resistance calculation were developed that account for the 3D shape of the droplet and the effect of the viscosity contrast between the two fluids. Finally, a modified pinchoff criterion was developed based on experimental observations that includes the shape of the gap and the strength of the Laplace pressure driven flow. The model captures the strong influence of the flow ratio, $\mathrm{Ca}$, and geometry on droplet formation. 
Good agreement was found between the model and experiments for the droplet size, frequency, and spacing across all the conditions considered, with most data falling within $10 \%$ of the predicted values. Additionally, $h^{*} / \mathrm{Ca}$ was identified as an important parameter for defining the flow in the squeezing-to-transition regime as it determines the degree of squeezing pressure created on the emerging droplet interface. Although the current model was validated with only liquid-liquid systems, it should translate just as well to gas-liquid systems.

Despite the success, more testing is required to define the applicability of the model. This includes extending the range of experiments into $\Lambda^{*}>1, \mathrm{Ca}>0.005$, and $\eta \gg 1$ to determine the limits of the assumptions that were applied. Future extensions of the model into the dripping regime must include the shear stress in the force balance. Still, in its current form, the model is sufficiently robust to enable the efficient design of T-junction generators without the ambiguity of trial and error methods. Other applications for the model may also include direct integration into real-time control systems or as a subroutine in a larger droplet network trafficking model $[18,19]$.

\section{ACKNOWLEDGMENTS}

The authors acknowledge the Natural Science and Engineering Research Council of Canada, Canada Research Chair program, Canada Foundation for Innovation, and University of Waterloo for research grants to C.R. and research support to C.E.; Ontarios's Early Research Award to C.R.; the Canada Graduate Scholarship to T.G.; and Danny Chan's assistance with the CFD models.
[1] T. Glawdel, C. Elbuken, and C. Ren, Phys. Rev. E 85, 016322 (2012).

[2] G. F. Christopher and S. L. Anna, J. Phys. D 40, R319 (2007).

[3] G. F. Christopher, N. N. Noharuddin, J. A. Taylor, and S. L. Anna, Phys. Rev. E 78, 036317 (2008).

[4] P. Garstecki, M. J. Fuerstman, H. A. Stone, and G. M. Whitesides, Lab. Chip. 6, 437 (2006).

[5] M. De Menech, P. Garstecki, F. Jousse, and H. A. Stone, J. Fluid. Mech. 595, 141 (2008).

[6] K. Wang, Y. C. Lu, J. H. Xu, and G. S. Luo, Langmuir 25, 2153 (2009).

[7] M. L. J. Steegmans, A. Warmerdam, K. Schroen, and R. M. Boom, Langmuir 25, 9751 (2009).

[8] K. J. Humphry, A. Ajdari, A. Fernandez-Nieves, H. A. Stone, and D. A. Weitz, Phys. Rev. E 79, 056310 (2009).

[9] S. van der Graaf, M. L. J. Steegmans, R. G. M. van der Sman, C. Schroen, and R. M. Boom, Colloids Surf. A 266, 106 (2005).
[10] V. van Steijn, C. R. Kleijn, and M. T. Kreutzer, Phys. Rev. Lett. 103, 214501 (2009).

[11] V. van Steijn, C. R. Kleijn, and M. T. Kreutzer, Lab. Chip. 10, 2513 (2010).

[12] http://mathworld.wolfram.com/CircularSegment.html.

[13] F. White, Fluid Mechanics, 5th ed. (McGraw-Hill, New York, 2002).

[14] S. W. Tchikanda, R. H. Nilson, and S. K. Griffiths, Int. J. Heat Mass Transfer 47, 527 (2004).

[15] H. A. Stone, Chem. Eng. Sci. 60, 4838 (2005).

[16] A. Gunther and K. F. Jensen, Lab. Chip. 6, 1487 (2006).

[17] See Supplemental Material at http://link.aps.org/supplemental/ 10.1103/PhysRevE.85.016323 for details regarding the compact listing of equations.

[18] F. Jousse, G. P. Lian, R. Janes, and J. Melrose, Lab. Chip. 5, 646 (2005).

[19] M. Schindler and A. Ajdari, Phys. Rev. Lett. 100, 044501 (2008). 